DESY 06-071

\title{
Non-thermal Production of Dark Matter from Late-Decaying Scalar Field at Intermediate Scale
}

\author{
Motoi Endo and Fuminobu Takahashi \\ Deutsches Elektronen Synchrotron DESY, \\ Notkestrasse 85, 22607 Hamburg, Germany \\ Institute for Cosmic Ray Research, \\ University of Tokyo, Chiba 27\%-8582, Japan
}

\begin{abstract}
We examine non-thermal dark matter production from a late-decaying scalar field, with a particular attention on non-renormalizable operators of $D=5$ through which the scalar field decays into the standard model particles and their superpartners. We show that almost the same number of superparticles as that of particles are generally produced from the decay. To avoid the gravitino overproduction problem, the decay is favored to proceed via interactions with an intermediate cut-off scale $M \ll M_{P}$. This should be contrasted to the conventional scenario using the modulus decay. The bosonic supersymmetry partner of the axion, i.e., saxion, is proposed as a natural candidate for such late-decaying scalar fields. We find that a right amount of the wino/higgsino dark matter with a mass of $O(100) \mathrm{GeV}$ is obtained for the saxion mass around the weak scale and axion decay constant, $F_{\mathcal{A}}=O\left(10^{9-12}\right) \mathrm{GeV}$.
\end{abstract}




\section{INTRODUCTION}

The lightest superparticle (LSP) is one of the most plausible candidates for the dark matter (DM) in supersymmetric (SUSY) models with $R$-parity conservation. In the SUSY standard model, the lightest neutralino, composed of superpartners of the gauge and Higgs bosons, is usually the LSP. From both the theoretical and experimental/observational points of view, the wino/higgsino DM is interesting, and it has been studied extensively so far 1 , 2, 3]. In this paper, we will concentrate on scenarios of the wino/higgsino DM production.

The present DM abundance is

$$
\Omega_{\mathrm{DM}} h^{2}=0.11 \pm 0.01
$$

from the latest WMAP three year data [4], where $h$ is the present Hubble parameter in units of $100 \mathrm{~km} / \mathrm{s} / \mathrm{Mpc}$. The relic abundance of the LSP must fall in this range to account for the DM abundance. The relic LSP abundance depends on the thermal history of the universe. Once the universe is reheated up to high temperature after inflation, the LSP is thermally produced by particle scatterings and reaches thermal equilibrium. However, it is known that the thermal processes cannot provide a sufficient amount of the wino/higgsino LSP with a mass, $m_{\chi}=O(100) \mathrm{GeV}$, because of the large annihilation rates. Therefore, non-thermal production must take place below the decoupling temperature, $T_{\chi} \sim m_{\chi} / 20$, to realize the observed DM abundance.

The non-thermal DM production at such a low temperature may be realized by a latetime decay of a scalar field, $\phi$, with a mass $m_{\phi}$. Renormalizable interactions generically induce a too rapid decay into the standard model (SM) particles and their superpartners unless the couplings are suppressed by some symmetries or mechanisms. Thus the decay should proceed via non-renormalizable operators suppressed by a large cut-off scale. In this paper, we focus on the $D=5$ operators. We will show that the branching ratio of the scalar decay into the SM superparticles is comparable to that into the SM particles.

A modulus is one of such late-time decaying fields [5], because the interactions of the modulus field are suppressed by the Planck scale, $M_{P}=2.4 \times 10^{18} \mathrm{GeV}$. The decay temperature of the modulus field is likely lower than the decoupling temperature, leading to the non-thermal production of the wino/higgsino LSP. However, according to the recent works [6, 7, 8, 9, 10], the late-time scalar decay is generically plagued with the gravitino 
overproduction. Thus the cosmological scenario with a modulus field is strongly constrained, for example, from the big-bang nucleosynthesis (BBN).

We point out that a natural source of the non-thermal DM production is related to new physics at an intermediate scale, $M \ll M_{P}$. The gravitino overproduction problem is then relaxed. This is because the total decay rate is enhanced as $\Gamma \sim m_{\phi}^{3} / M^{2}$, reducing the branching ratio of the gravitino production. Note also that, since a light scalar field becomes acceptable, the decay into the gravitinos may be kinematically forbidden. In the following sections we will show that a right amount of the wino/higgsino DM is obtained by the scalar decay at an intermediate scale. We propose the saxion field, which is the bosonic SUSY partner of the axion, as a natural candidate for such a scalar field.

Before closing the introduction, let us clarify differences from the works in the past, which discussed the non-thermal DM production from late-time decaying scalar fields. The decay from a heavy scalar with Planck-suppressed couplings, i.e., a modulus field, has been extensively discussed in Ref. [5]. Also, Ref. [11] analyzed a similar subject with a lower cutoff, although they regarded the branching ratios of the superparticle as a free parameter. In this paper, we explicitly show that the branching ratio of the SM superparticle production is generically large, and that the effective number of the LSP produced by one scalar decay, $N_{\chi}$, is close to unity. This has a great impact on the non-thermal DM production scenarios.

The rest of the paper is organized as follows. In Sec. II we estimate the branching fraction of the SM (super)particle production for the scalar decay through the non-renormalizable operators of $D=5$. We reexamine the conventional scenario using the modulus decay for the non-thermal DM production in Sec. III It is shown that the saxion decay can account for the present DM abundance in Sec. IV] The last section is devoted to conclusion.

\section{HEAVY SCALAR DECAY VIA $D=5$ OPERATORS}

In this section we consider a heavy scalar decay into the SM particles and their superpartners, assuming the scalar mass $m_{\phi}$ much larger than the weak scale. Since those particles with R-parity odd eventually produce at least one LSP per decay, the production rate of the superparticles is crucial to determine the relic LSP abundance. In the following, we assume that the scalar field is R-parity even and singlet under the SM gauge groups. The purpose of this section is to estimate the decay rates of the scalar field into the SM (super)particles 
via the non-renormalizable operators of $D=5$. Although such operators have been studied in Ref. [5], they overlooked some important interactions of the superparticle production. Below we investigate the operators one by one.

(I) Let us first consider the scalar decay into the gauge boson and gaugino. Such an interaction is effectively given by the dilatonic coupling with a cut-off scale $M$,

$$
\mathcal{L}=\frac{\lambda_{G}}{M} \int d^{2} \theta \phi W^{(a)} W^{(a)},
$$

where $\lambda_{G}$ is a numerical coefficient and $W^{(a)}$ is the supersymmetric field strength of the SM gauge supermultiplet. Including the supergravity effects, we obtain

$$
\begin{aligned}
\mathcal{L}_{G} \simeq & \frac{\lambda_{G}}{M}\left[\frac{\phi_{R}}{\sqrt{2}}\left(-\frac{1}{4} F_{\mu \nu}^{(a)} F^{(a) \mu \nu}\right)+\frac{\phi_{I}}{\sqrt{2}}\left(-\frac{1}{8} \epsilon^{\mu \nu \rho \sigma} F_{\mu \nu}^{(a)} F_{\rho \sigma}^{(a)}\right)\right. \\
& \left.+\frac{1}{4} e^{G / 2}\left\{\left(G^{\bar{\phi}}{ }_{\phi} \phi+G^{\bar{\phi}}{ }_{\bar{\phi}} \phi^{\dagger}\right) \bar{\lambda}^{(a)} \mathcal{P}_{R} \lambda^{(a)}+\left(G^{\phi}{ }_{\bar{\phi}} \phi^{\dagger}+G_{\phi}^{\phi} \phi\right) \bar{\lambda}^{(a)} \mathcal{P}_{L} \lambda^{(a)}\right\}\right],
\end{aligned}
$$

where the real and imaginary components of the scalar field are defined as $\phi \equiv\left(\phi_{R}+i \phi_{I}\right) / \sqrt{2}$. Although there are the other contributions from the gaugino kinetic terms, the resultant decay rate through them is proportional to the gaugino mass squared, $m_{\lambda}^{2}$, after applying the equation of motion. Therefore they are neglected unless $m_{\phi}$ is close to (but larger than) $2 m_{\lambda}$. The subindex, $i$, attached to the total Kähler potential, $G=K+\ln |W|^{2}$, represents a derivative with respect to the field, $i$, while the superscript is obtained by $G^{i}=g^{i j^{*}} G_{j^{*}}$, where $g^{i j^{*}}$ is the inverse of the Kähler metric $g_{i j^{*}}$. The coefficient $e^{G / 2}$ is equal to the gravitino mass at the vacuum, $m_{3 / 2}=\left\langle e^{G / 2}\right\rangle$.

The coefficients of the interactions with the gauginos are

$$
\begin{aligned}
G_{\bar{\phi}}^{\phi} & =\left(g^{\phi \bar{\phi}} G_{\bar{\phi}}\right)_{\bar{\phi}}=g^{\phi \bar{\phi}} G_{\bar{\phi} \bar{\phi}}+\left(g^{\phi \bar{\phi}}\right)_{\bar{\phi}} G_{\bar{\phi}}, \\
G_{\phi}^{\phi} & =\left(g^{\phi \bar{\phi}} G_{\bar{\phi}}\right)_{\phi}=1+\left(g^{\phi \bar{\phi}}\right)_{\phi} G_{\bar{\phi}}
\end{aligned}
$$

where we have assumed $g_{i j^{*}}=0$ for $i \neq j$, for simplicity. If $m_{\phi} \lesssim m_{3 / 2}$, we can see $G^{\phi}{ }_{\bar{\phi}}+$ $G^{\phi}{ }_{\phi}=O(1)$ barring cancellations. In fact, the minimal Kähler potential without the SUSY mass term (i.e., $G_{\phi \phi}=0$ ) gives $G^{\phi}{ }_{\bar{\phi}}+G_{\phi}^{\phi}=1$. On the other hand, if the mass is enhanced by the SUSY mass, namely $m_{\phi} \simeq e^{G / 2}\left|G_{\phi \phi}\right| \gg m_{3 / 2}$, the coefficient is enhanced as $G^{\phi}{ }_{\bar{\phi}}+G_{\phi}^{\phi}=$ $O\left(m_{\phi} / m_{3 / 2}\right)$. However if the scalar mass $m_{\phi}$ is enhanced due to the non-SUSY effects, e.g., $\delta K=|\phi|^{2}|z|^{2} / M^{2}$ with the SUSY breaking field $z$, the coupling is given by $G_{{ }_{\phi}}+G_{\phi}^{\phi}=O(1)$.

Then the decay rates into the gauge multiplets are evaluated as [6]

$$
\Gamma_{G} \equiv \Gamma(\phi \rightarrow \text { gauge boson }) \simeq \Gamma(\phi \rightarrow \text { gaugino }) \simeq N_{g} \frac{\left|\lambda_{G}\right|^{2}}{8 \pi} \frac{m_{\phi}^{3}}{M^{2}},
$$


either if $m_{\phi} \sim m_{3 / 2}$ or if $m_{\phi} \gg m_{3 / 2}$ due to the SUSY mass term. Here $N_{g}$ is the number of the possible final states, and $N_{g}=12$ for the SM gauge groups, $S U(3)_{C} \times S U(2)_{L} \times U(1)_{Y}$. We find that the production rate of the gaugino is comparable to that of the gauge boson, and is not suppressed by the gaugino mass. On the other hand, if the scalar mass is smaller than $m_{3 / 2}$, the gaugino production rate is estimated as $\Gamma \sim m_{3 / 2}^{2} m_{\phi} / M^{2}$ up to a numerical factor, while the gauge boson production rate is the same as (6). Similarly, if $m_{\phi}$ is enhanced due to the non-SUSY mass term as $m_{\phi} \gg m_{3 / 2}$, the gaugino production rate is $\Gamma \sim m_{3 / 2}^{2} m_{\phi} / M^{2}$, which is suppressed compared to the gauge boson production rate $\Gamma \sim m_{\phi}^{3} / M^{2}$.

(II) The scalar field can couple with the matter fields in the Kähler potential. When the Kähler potential includes such a non-renormalizable term as, $K=\phi Q Q^{\prime \dagger} / M+h . c$. , in which the matter fields $Q$ and $Q^{\prime}$ appear with the opposite chiralities, we can show that the decay rate into the SM (super)particle is proportional to powers of the masses of the matter fields.

Let us first consider the matter scalar production rate. The first contribution comes from the kinetic term of the scalar fields: $\mathcal{L}_{K}=g_{i j^{*}} \partial_{\mu} \phi^{i} \partial^{\mu} \phi^{j *}$. Then the decay rate is proportional to the fourth power of the mass of the matter scalar after using the equation of motion, since the interaction term becomes $\mathcal{L}_{K}=-(\phi / M) \tilde{Q}\left(\partial^{2} \tilde{Q}^{\prime}\right)$ up to a total derivative term, where $\tilde{Q}$ is the scalar component of the matter chiral multiplet $Q[5]$. Thus the decay rate is estimated as $\Gamma_{K} \sim\left(m_{Q^{\prime}} / m_{\phi}\right)^{4} \times m_{\phi}^{3} / M^{2}$.

The other couplings arise from the scalar potential. In particular, the decay rate may be enhanced due to the supergravity effects when $m_{3 / 2}$ is large. Since non-renormalizable operators violate the conformal symmetry, the operator receives corrections of order $m_{3 / 2}^{2}$ at the leading level. Thus we obtain $\mathcal{L}_{V} \sim \frac{1}{M} m_{3 / 2}^{2} \phi \tilde{Q} \tilde{Q}^{\prime \dagger}+$ h.c., though the coefficient depends on details of the models. The decay rate then becomes $\Gamma_{V} \sim\left(m_{3 / 2} / m_{\phi}\right)^{4} \times m_{\phi}^{3} / M^{2}$.

The above supergravity effects seem to give a correction to the matter scalar mass, $\delta m_{Q}^{2} \sim$ $m_{3 / 2}^{2}$, by taking a vacuum expectation value (VEV), $\langle\phi\rangle \sim M$. It should be noted, however, that this estimate is too naive and actually overestimates the correction when $m_{3 / 2}$ is larger than the weak scale. This is because there exists a cancellation among the contributions to the soft scalar mass if $\left|G_{\phi}\right| \ll\langle\phi\rangle$. This cancellation can be understood in terms of the Kähler invariance. To be concrete, let us consider the minimal Kähler potential with the coupling $\delta K=\phi Q Q^{\prime \dagger} / M+h . c$. . Since the above contribution in $O\left(m_{3 / 2}^{2}\right)$ arises from $K_{\phi} W$ of the scalar potential, it is always accompanied by $W_{\phi}$. Thus when the F-term of $\phi$ is suppressed, the correction of $O\left(m_{3 / 2}^{2}\right)$ will cancel with that from $W_{\phi}$ after taking the VEV. 
Lastly, the production rates of the matter fermions, $\psi_{Q}$, are chirally suppressed. The decay rate is estimated as $\Gamma_{\psi_{Q}} \sim\left(m_{\psi_{Q}} / m_{\phi}\right)^{2} \times m_{\phi}^{3} / M^{2}$, which is proportional to the fermion mass squared.

(III) The heavy scalar field can decay into the Higgs supermultiplets via the interaction:

$$
\mathcal{L}=\frac{\lambda_{H}}{M} \int d^{4} \theta \phi^{\dagger} H_{u} H_{d}+\text { h.c. }
$$

though such a coupling is related to the $\mu$-problem and it is quite model-dependent whether or not such a coupling exists. We notice that the Higgs chiral supermultiplets appear with the same chirality. Expanding the interaction (7), we obtain

$$
\mathcal{L}_{H} \simeq \frac{\lambda_{H}}{M}\left[-\left(\partial^{2} \phi^{\dagger}\right) H_{u} H_{d}+e^{G / 2} G^{\bar{\phi}} \phi \phi \overline{\tilde{H}}_{u R} \tilde{H}_{d L}\right]+\text { h.c.. }
$$

In addition, the scalar trilinear coupling $\phi^{\dagger} H_{u} H_{d}$ arises from the scalar potential; the decay rate through the supergravity corrections is given by $\sim\left|\lambda_{H}\right|^{2} m_{3 / 2}^{2} m_{\phi} / M^{2}$. We neglect the interaction terms whose coupling is suppressed by the higgsino mass. In particular, they include the $U(1)$ connection term in the covariant derivative of the higgsino kinetic term, and that obtained by expanding $e^{G / 2}$ in the higgsino mass term. These are generically small in the mass eigenstate basis by considering the interference with the SUSY breaking field $[9,10]$.

Thus, either if $m_{\phi} \sim m_{3 / 2}$ or if $m_{\phi} \gg m_{3 / 2}$ due to the SUSY mass term, the decay rates are given by

$$
\Gamma(\phi \rightarrow H H) \simeq \Gamma(\phi \rightarrow \tilde{H} \tilde{H}) \simeq \frac{\left|\lambda_{H}\right|^{2}}{8 \pi} \frac{m_{\phi}^{3}}{M^{2}}
$$

for the Higgs multiplets which are doublet under the $S U(2)_{L}$ symmetry. We find that the decay rates are comparable to $\Gamma_{G}$ if $\lambda_{G} \sim \lambda_{H}$, and that the higgsino production rate is the same order of the Higgs production rate. In contrast, when the scalar mass is enhanced by the non-SUSY mass term as $m_{\phi} \gg m_{3 / 2}$, the higgsino production is suppressed compared to the decay into the Higgs bosons by $O\left(m_{3 / 2}^{2} / m_{\phi}^{2}\right)$.

(IV)The following interactions in the superpotential permit the three-body decay,

$$
\mathcal{L}=\frac{\lambda_{Y}}{M} \int d^{2} \theta \phi H_{u} T^{c} Q+\text { h.c. }
$$

where $H_{u}, T, Q$ are the chiral supermultiplet of the up-type Higgs, right-handed top quark and left-handed quark doublet of the third generation, respectively. Then the interactions 
become

$$
\mathcal{L}_{Y}=\frac{\lambda_{Y}}{M}\left[\phi H_{u} \bar{t} q+\phi \tilde{t}^{*} \overline{\tilde{H}}_{u R} q+\phi \bar{t} \tilde{H}_{u L} \tilde{q}\right]+\text { h.c. }
$$

in the global SUSY Lagrangian. Although the decay rates do not receive chirality suppressions, the decay is a three-body process and the phase space is small [5]:

$$
\Gamma(\phi \rightarrow H t q) \simeq \Gamma(\phi \rightarrow \tilde{H} \tilde{t} q) \simeq \Gamma(\phi \rightarrow \tilde{H} t \tilde{q}) \simeq \frac{\left|\lambda_{Y}\right|^{2}}{256 \pi^{3}} \frac{m_{\phi}^{3}}{M^{2}}
$$

for $m_{\phi} \gg m_{\text {soft }}$. Note that couplings with the lighter quarks and leptons must be suppressed, since the corresponding Yukawa coupling constants need fine-tunings by taking account of the scalar VEV, $\langle\phi\rangle \sim M$.

Additionally, the scalar couplings receive supergravity corrections. The decay rate through the correction which is of order $m_{3 / 2}$ for the operator $\phi H_{u} \tilde{u}^{*} \tilde{q}$ is suppressed both by the phase space and by the gravitino mass squared. If $m_{\phi}<m_{3 / 2}$, the decay rate becomes larger than (12). At first sight, the scalar VEV seems to induce a correction of an order $m_{3 / 2}$ to the trilinear scalar coupling, $H_{u} \tilde{u}^{*} \tilde{q}$. However, it is actually suppressed if $\left|G_{\phi}\right| \ll\langle\phi\rangle$, since the scalar $\phi$ contributes to the A-parameter through the auxiliary component $G_{\phi}$ due to the Kähler invariance.

From the analyses of (I) - (IV), either if $m_{\phi} \sim m_{3 / 2}$ or if $m_{\phi} \gg m_{3 / 2}$ due to the SUSY mass $G_{\phi \phi} \gg 1$, almost the same amount of the SM superparticles is produced as that of the SM particles:

$$
\operatorname{Br}(\phi \rightarrow \text { SM particles }) \simeq \operatorname{Br}(\phi \rightarrow \text { SM superparticles })
$$

with the total decay rate

$$
\Gamma_{\phi}=\frac{c}{4 \pi} \frac{m_{\phi}^{3}}{M^{2}}
$$

where $c$ is a numerical coefficient, and given by $c \simeq N_{g} \lambda_{G}^{2}+\lambda_{H}^{2}$, for the dilatonic coupling and the interaction with the Higgs supermultiplets. The other decay processes are suppressed either by the soft masses or by the phase space. On the other hand, when the scalar mass is enhanced by the non-SUSY mass term as $m_{\phi} \gg m_{3 / 2}$, the above superparticle production rate becomes suppressed compared to that of the SM particle. Note that the single-gravitino production induce a serious problem in this case, as will be discussed in the 
next section. If $m_{3 / 2}>m_{\phi}$, the supergravity corrections significantly affect the decay rates, and the branching ratio of the superparticle production depends on the non-renormalizable operators. We emphasize again that the branching ratio of the superparticle production is generically sizable, $\operatorname{Br}(\phi \rightarrow \mathrm{SM}$ superparticles $)=O(0.1)$, either when $m_{\phi} \sim m_{3 / 2}$ or if $m_{\phi} \gg m_{3 / 2}$ due to the SUSY mass term.

\section{DIFFICULTIES IN MODULUS DECAY}

It has been known that the wino/higgsino LSP with a mass of $O(100) \mathrm{GeV}$ cannot be produced sufficiently in the thermal bath because of its large annihilation cross section. We need therefore the non-thermal process below the freeze-out temperature in order to explain the observed DM abundance. In this section let us reexamine the non-thermal DM production from the modulus decay.

The modulus field corresponds to a dynamical degree of freedom associated with a flat direction of the scalar potential. Since the SUSY breaking effects stabilize the potential, the modulus field naturally obtains a mass around the gravitino mass, $m_{3 / 2}$. In addition, the modulus mass can be even larger with sizable non-perturbative corrections. The modulus decay is generally induced by the non-renormalizable operators, whose couplings are suppressed by the Planck scale, namely $M=M_{P}$ in the previous section. Such a light and late-decaying scalar field is likely to dominate the energy of the universe before its decay. Then the subsequent thermal history depends on the decay processes of the modulus field, that is, the decay temperature and the branching fractions of the decay products. The modulus mass is required to be $m_{\phi} \gtrsim 100 \mathrm{TeV}[5]$ a , from the cosmological BBN bound.

If the decay takes place after the freeze-out of the LSP, the relic LSP abundance is estimated in terms of the following parameters: the annihilation cross section of the LSP, $\langle\sigma v\rangle$, the modulus decay temperature, $T_{d}$, and the branching fraction of the superparticle production, which provides an effective number of the LSP from one modulus decay, $N_{\chi}$. According to the previous section, the branching fraction of the LSP production is generically quite large. Then the resultant LSP abundance after the pair annihilation becomes independent

\footnotetext{
${ }^{a}$ One might consider the modulus decay via the $D=6$ operators. However it is unlikely that the decay takes place before the BBN starts.
} 
of the initial abundance, and given by 12 ]

$$
Y_{\mathrm{LSP}} \simeq \sqrt{\frac{45}{8 \pi^{2} g_{* d}}} \frac{1}{\langle\sigma v\rangle M_{P} T_{d}},
$$

or equivalently, the relic LSP density is

$$
\Omega_{\chi} h^{2} \simeq 0.5\left(\frac{10.75}{g_{* d}}\right)^{\frac{1}{2}}\left(\frac{10^{-7} \mathrm{GeV}^{-2}}{\langle\sigma v\rangle}\right)\left(\frac{m_{\chi}}{100 \mathrm{GeV}}\right)\left(\frac{100 \mathrm{MeV}}{T_{d}}\right)
$$

where $m_{\chi}$ is the LSP mass. In the case of the wino LSP, the annihilation cross section is estimated to be e.g. $\langle\sigma v\rangle \simeq 3.3 \times 10^{-7} \mathrm{GeV}^{-2}$ for the wino mass $M_{2}=100 \mathrm{GeV}$, and that of the higgsino LSP is roughly the same order of the magnitude (see Eqs. (32) and (333)). Since the annihilation cross section decreases for the larger LSP mass, it is likely smaller than $O\left(10^{-7}\right) \mathrm{GeV}^{-2}$. Thus the decay temperature must satisfy $T_{d} \gtrsim 100 \mathrm{MeV}$ in order to obtain a right amount of the relic DM abundance (11).

Using the total decay rate (14) with $M=M_{P}$, the decay temperature is given by

$$
T_{d} \simeq 5.5 \mathrm{MeV} \cdot c^{\frac{1}{2}}\left(\frac{m_{\phi}}{100 \mathrm{TeV}}\right)^{3 / 2}
$$

where $c=O(1)$. To satisfy $T_{d} \gtrsim 100 \mathrm{MeV}$, the modulus mass should be large enough:

$$
m_{\phi} \gtrsim 10^{6-7} \mathrm{GeV}
$$

The bound on the modulus mass becomes severer for smaller $c$ and the heavier wino/higgsino. It should be stressed again that one of the crucial points to derive the above constraint is an effective number of the LSP from the modulus decay. As long as the decay proceeds through the non-renormalizable operators of $D=5$, the branching ratio of the LSP production is generically large, and close to unity. This is another difficulty of the modulus decay, and let us call it as "the moduli-induced LSP problem".

The mass of the modulus field depends on the mechanism of the potential stabilization. A modulus field as heavy as $m_{\phi} \gtrsim 10^{6-7} \mathrm{GeV}$ may be realized in the scenarios of the mixed modulus-anomaly/KKLT mediation [2] and in the racetrack setups 13]. However, such a heavy modulus generically suffers from the gravitino overproduction [6]. Since the conformal anomaly mediates the SUSY breaking effects to the visible sector and the correction is related to the gravitino mass [1], the gravitino mass is favored to be $\lesssim 100 \mathrm{TeV}$, leading to the relation $m_{\phi}>2 m_{3 / 2}$. The production rate of the goldstino, which is a longitudinal 
component of the gravitino, can be enhanced in the mode $\phi \rightarrow 2 \psi_{3 / 2}$ in high energy limit $p_{\mu} \gg m_{3 / 2}$. Actually, the decay rate is obtained as $[6]$

$$
\Gamma\left(\tilde{\Phi} \rightarrow 2 \psi_{3 / 2}\right) \simeq \frac{\left|\mathcal{G}_{\Phi}^{(\mathrm{eff})}\right|^{2}}{288 \pi} \frac{m_{\phi}^{5}}{m_{3 / 2}^{2} M_{P}^{2}}
$$

where $\tilde{\Phi}$ denotes the mass eigenstate (mainly composed of $\phi$ ), and $\mathcal{G}_{\Phi}^{(\text {eff })}$ is the effective auxiliary field of $\tilde{\Phi}$. The auxiliary component of the scalar field crucially depends on the structure of the SUSY breaking sector as was pointed out in Ref. [9]. Since both the modulus and SUSY breaking fields are not generally protected by any symmetries at the vacuum, they couple with each other, and the rate becomes sizable ${ }^{b}$. In fact, the effective auxiliary field can be as large as $\mathcal{G}_{\Phi}^{(\text {eff })}=\kappa_{3 / 2} m_{3 / 2} / m_{\phi}$ with $\kappa_{3 / 2}=O(1)$ [10]. Thus the branching ratio of the gravitino production is $B_{3 / 2}=O(0.01-0.1)$. Such models with a large gravitino production is severely constrained by the cosmology, which we call "the moduli-induced gravitino problem" [6] .

As a final remark of this section, let us mention the case of a modulus mass coming from the non-SUSY mass which satisfies $m_{\phi} \gg m_{3 / 2}$. In this case, a mass of the fermionic component of $\phi$ is likely much smaller than $m_{\phi}$, so the single-gravitino production starts to dominate over the pair production. The decay rate is [15]

$$
\Gamma\left(\phi \rightarrow \psi_{\phi} \psi_{3 / 2}\right) \simeq \frac{1}{48 \pi} \frac{m_{\phi}^{5}}{m_{3 / 2}^{2} M_{P}^{2}}
$$

for $m_{\phi} \gg m_{\psi_{\phi}}, m_{3 / 2}$, which dominates over the other decay processes. It is noticed that the rate is independent of the VEV of the effective auxiliary field $\mathcal{G}_{\Phi}^{(\text {eff })}$. Thus such modulus field generally suffers from the production of too much gravitinos.

In summary, the modulus decay is disfavored as a source of the non-thermal DM production because of the above "the moduli-induced gravitino/LSP problems." In the next section, we propose a natural solution which is free from these problems and also explains the relic wino/higgsino DM abundance.

\footnotetext{
${ }^{\mathrm{b}}$ Even if the modulus does not couple to the SUSY breaking sector, the branching fraction of the gravitino production is still large. If the scalar mass of the SUSY breaking field, $m_{z}$, is smaller than $m_{\phi}$ as $B_{3 / 2}=O\left(10^{-4}-10^{-3}\right)$, while $B_{3 / 2}=O(0.01-0.1)$ if $m_{z}>m_{\phi}$. Such large branching ratio causes serious cosmological problem [14].
} 


\section{DARK MATTER FROM SAXION DECAY}

The difficulties of the modulus decay in producing a right amount of DM are associated with the fact that the interactions are suppressed by the Planck scale, as shown in the previous section. The modulus mass cannot be smaller than $100 \mathrm{TeV}$ to decay before BBN, which makes the modulus decay into the gravitinos almost inevitable. One of the attractive ways to get around the problem is to postulate a cut-off scale smaller than the Planck scale; the branching ratio of the gravitino production can be then suppressed, or may even be kinematically forbidden. Thus an intermediate-scale physics is likely to play an important role in the non-thermal wino/higgsino DM production.

An intermediate-scale physics naturally arises in the Pecci-Quinn (PQ) mechanism 16, 17, 18, 19]. The strong CP problem is one of the profound puzzles in SM, and the PQ mechanism using a pseudo Nambu-Goldstone boson called axion provides an elegant solution to the problem. The axion field has a flat potential at a perturbative level, and acquires a finite mass from non-perturbative QCD effects via the anomaly. The axion rolls down to the potential minimum at which the strong CP phase vanishes. From the cosmological/astrophysical observations, the axion decay constant, $F_{\mathcal{A}}$, is required to satisfy, $F_{\mathcal{A}} \gtrsim 10^{9} \mathrm{GeV}$ from SN1987a [20, 21], and $F_{\mathcal{A}} \lesssim 10^{12-15} \mathrm{GeV}$ by the axion-overclosure limit ${ }^{\mathrm{c}}$, although the latter depends on the cosmological scenario [22, 23, 24].

In the SUSY theories, the axion field is extended to the axion supermultiplet, $\mathcal{A}$. The multiplet contains two bosonic real fields, which are axion and saxion, and one chiral fermion called axino. The scalar component of the axion multiplet is represented as

$$
\mathcal{A} \equiv \frac{1}{\sqrt{2}}(\sigma+i a)
$$

where $\sigma$ and $a$ are saxion and axion fields, respectively. The saxion behaves like a modulus in the evolution of the universe. Since the saxion potential as well is flat at a perturbative level in the SUSY limit, the field is stabilized by the SUSY breaking (and perhaps nonperturbative corrections), and it acquires a mass around the gravitino mass, $m_{\sigma} \sim m_{3 / 2}{ }^{\mathrm{d}}$. The initial position of the saxion generically deviates from the potential minimum in the

\footnotetext{
${ }^{c}$ As is well known, the axion itself is a DM candidate. However the contribution depends on the initial condition of the axion field and the subsequent thermal history. In the following, we will focus on the abundance of the wino/higgsino LSP.

${ }^{\mathrm{d}}$ Precisely speaking, the saxion mass is model-dependent. For instance, in the heavy gravitino setups, if
} 
early universe, and it starts to oscillate when the Hubble parameter becomes comparable to the saxion mass. Then the energy fraction of the saxion can be sizable, and large number of the superparticles may be produced at the decay ${ }^{\mathrm{e}}$.

It is a strength of the couplings with the SM sector that significantly differs from the modulus field. The axion supermultiplet interacts with the SM (super)particles with the couplings suppressed by the axion decay constant, $F_{\mathcal{A}}$. Since $F_{\mathcal{A}}$ must be much smaller than the Planck scale because of the cosmological requirement, the saxion decay rate is much larger than that of the modulus field with the same mass. In particular, the axion multiplet $\mathcal{A}$ necessarily has a dilatonic coupling:

$$
\mathcal{L}=-\int d^{2} \theta \frac{\alpha}{4 \sqrt{2} \pi} \frac{\mathcal{A}}{F_{\mathcal{A}}} W^{(a)} W^{(a)},
$$

where $\alpha$ is a gauge coupling constant, and $F_{\mathcal{A}}$ the axion decay constant. Here we adopt the hadronic axion model [18] for simplicity, and a brief comment on the case of the DFSZ axion model [19] will be given later. The dilatonic coupling arises from the chiral anomaly of the PQ symmetry which is extended to the complex $U(1)_{P Q}$ symmetry in the SUSY framework. The interaction (22) actually reproduces the axion coupling with the $S U(3)_{C}$ gauge bosons, providing a solution to the strong CP problem. It also provides the saxion couplings with the gauge bosons and gauginos:

$$
\mathcal{L}=\frac{\alpha}{8 \pi} \frac{\sigma}{F_{\mathcal{A}}} F_{\mu \nu}^{(a)} F^{(a) \mu \nu}-\frac{\alpha}{8 \pi} \frac{\sigma}{F_{\mathcal{A}}} e^{G / 2}\left[\left(G_{A}^{A}+G_{\bar{A}}^{A}\right) \bar{\lambda}^{(a)} \mathcal{P}_{L} \lambda^{(a)}+\text { h.c. }\right]
$$

where $F_{\mu \nu}^{(a)}$ and $\lambda^{(a)}$ denote the field strength of the gauge boson and the gaugino field, respectively. The decay rates of the saxion into the gauge bosons $g$ and gauginos $\lambda$ are given by ${ }^{\mathrm{f}}$

$$
\Gamma(\sigma \rightarrow g g) \simeq \frac{3 \alpha^{2}}{64 \pi^{3}} \frac{m_{\sigma}^{3}}{F_{\mathcal{A}}^{2}}, \quad \Gamma(\sigma \rightarrow \lambda \lambda) \simeq \frac{3 \alpha^{2}}{64 \pi^{3}}|\kappa|^{2} \frac{m_{\sigma}^{3}}{F_{\mathcal{A}}^{2}}
$$

the axion multiplet is sequestered from the SUSY breaking sector, the saxion mass can be smaller than the gravitino mass by a loop factor.

e The LSP may be the fermionic superpartner of the axion, axino. However the axino mass depends on the models, and we assume that the wino/higgsino is the LSP in the following.

${ }^{\mathrm{f}}$ The saxion may have a coupling with the axion, which enables the saxion to decay into two axions. If the saxion dominantly decays into the axions, it can be cosmologically problematic 25]. However, it is model-dependent whether this process dominates the saxion decay. In fact, such a coupling can be suppressed. In the following, we simply assume that the decay mode is suppressed. 
with

$$
\kappa \equiv\left(G_{A}^{A}+G_{\bar{A}}^{A}\right) \frac{m_{3 / 2}}{m_{\sigma}}
$$

where we have neglected the sub-leading terms suppressed by $\left(m_{\lambda} / m_{\sigma}\right)^{2}\left(m_{\lambda}\right.$ : the gaugino mass). Although we have assumed that the saxion universally couples to the $S U(3)_{C} \times$ $S U(2)_{L} \times U(1)_{Y}$ gauge multiplets in estimating the decay rates, this assumption is not crucial for the following arguments. This is because the saxion decay into the gauge bosons is dominated by that into gluons, to which the QCD axion multiplet necessarily couples. Thus the branching ratio of the gaugino production is $\operatorname{Br}(\sigma \rightarrow \lambda \lambda) \simeq|\kappa|^{2} /\left(1+|\kappa|^{2}\right)$, which depends on $\kappa$. As discussed in Sec. III $G_{A}^{A}+G^{A}{ }_{\bar{A}}$ is generically $O(1)$ for $m_{\sigma} \lesssim m_{3 / 2}$, leading to $\kappa=O(1)$. In fact, for the minimal Kähler potential we have $\left(G_{A}^{A}+G_{\bar{A}}^{A}\right)=1$. Here it should be note that the axion multiplet is not allowed to have nonzero SUSY mass, since the potential minimum of the axion would be shifted otherwise. On the other hand, if a mass hierarchy, $m_{\sigma} \gg m_{3 / 2}$, is realized, $\kappa$ can be suppressed as $O\left(m_{3 / 2} / m_{\sigma}\right)$. Thus the branching ratio of the gaugino production is sizable in the saxion decay,

$$
\operatorname{Br}(\sigma \rightarrow \lambda \lambda)=\frac{|\kappa|^{2}}{1+|\kappa|^{2}}=O(0.1)
$$

unless the saxion mass is much larger than $m_{3 / 2}$ due to the non-SUSY mass. It should be stressed that the branching ratio of the gaugino production is not suppressed by the gaugino mass as noted in the previous section, and that the decay rates are enhanced by $O\left(M_{P}^{2} / F_{\mathcal{A}}^{2}\right)$ compared to the modulus case.

The relic wino/higgsino LSP abundance is determined by solving the Boltzmann equations. The wino/higgsino LSP can be produced from thermal scatterings, but it is known that the thermal relic abundance is too small to account for the present DM density. As will be shown below, the saxion decay can non-thermally produce a large enough number of LSPs, due to both large branching ratio of the gaugino production and relatively low decay temperature.

The LSP number density $n_{\chi}$ evolves as

$$
\dot{n_{\chi}}+3 H n_{\chi}=-\langle\sigma v\rangle n_{\chi}^{2}
$$

where we have neglected the production of the LSPs from thermal scattering processes by assuming that the saxion decay temperature is lower than the decoupling temperature of 
the LSP, $T_{\chi} \sim m_{\chi} / 20$. Here $\langle\sigma v\rangle$ is the thermally averaged annihilation cross section of the LSP. Let us rewrite Eq. (27) in terms of the LSP abundance, $Y_{\chi} \equiv n_{\chi} / s$,

$$
\frac{d Y_{\chi}}{d T}=\sqrt{\frac{8 \pi^{2} g_{* d}}{45}}\langle\sigma v\rangle M_{P} Y_{\chi}^{2},
$$

where $g_{* d}$ is an effective number of massless degrees of freedom at the saxion decay, and we have approximated that $g_{* d}$ is almost constant during the evolution. By integrating this equation, we obtain the simple analytic formula of the relic abundance for $T<T_{d}$ [12]:

$$
Y_{\chi}(T)=\left[Y_{\chi}\left(T_{d}\right)^{-1}+\sqrt{\frac{8 \pi^{2} g_{* d}}{45}}\langle\sigma v\rangle M_{P}\left(T_{d}-T\right)\right]^{-1} .
$$

If the initial LSP abundance $Y_{\chi}\left(T_{d}\right)$ satisfies

$$
\begin{aligned}
Y_{\chi}\left(T_{d}\right) & \gg Y_{\chi}^{(c)} \equiv\left[\sqrt{\frac{8 \pi^{2} g_{* d}}{45}}\langle\sigma v\rangle M_{P} T_{d}\right]^{-1} \\
& \simeq 8.4 \times 10^{-12} \xi^{-1}\left(\frac{10.75}{g_{* d}}\right)^{\frac{1}{4}}\left(\frac{10^{-7} \mathrm{GeV}^{-2}}{\langle\sigma v\rangle}\right)\left(\frac{1 \mathrm{TeV}}{m_{\sigma}}\right)^{\frac{3}{2}}\left(\frac{F_{\mathcal{A}}}{10^{12} \mathrm{GeV}}\right),
\end{aligned}
$$

the second term in the parenthesis of Eq. (29) dominates over the first term. Then the final abundance is approximately given by

$$
Y_{\chi}^{(f)} \simeq \sqrt{\frac{45}{8 \pi^{2} g_{* d}}} \frac{1}{\langle\sigma v\rangle M_{P} T_{d}} .
$$

Therefore the relic abundance is determined only by the annihilation cross section of the LSP and the decay temperature of the parent particle, independent of the initial amount of the LSP.

The annihilation cross section, $\langle\sigma v\rangle$, for the wino LSP is evaluated as 5$]$

$$
\langle\sigma v\rangle \simeq \frac{g_{2}^{4}}{2 \pi} m_{\chi}^{-2} \frac{\left(1-x_{W}\right)^{3 / 2}}{\left(2-x_{W}\right)^{2}}
$$

where $x_{W} \equiv m_{W}^{2} / m_{\chi}^{2}$ denotes the mass squared ratio of the $\mathrm{W}$ boson and the $\operatorname{LSP}$ (wino), and $g_{2}$ is the gauge coupling constant of $S U(2)_{L}$. For $m_{\chi}=O\left(10^{2}\right) \mathrm{GeV},\langle\sigma v\rangle$ is roughly estimated as $O\left(10^{-7}\right) \mathrm{GeV}^{-2}$. For the higgsino LSP, it is given by [26]

$$
\langle\sigma v\rangle \simeq \frac{g_{2}^{4}}{32 \pi} m_{\chi}^{-2} \frac{\left(1-x_{W}\right)^{3 / 2}}{\left(2-x_{W}\right)^{2}}
$$

which is smaller than the wino cross section by one order of magnitude. 
Assuming that the saxion dominantly decays into the gauge bosons and gauginos with the rates given by Eq. (24), the decay temperature is given by

$$
T_{d} \simeq 1.1 \times 10^{2} \mathrm{MeV} \xi\left(\frac{10.75}{g_{* d}}\right)^{\frac{1}{4}}\left(\frac{m_{\sigma}}{1 \mathrm{TeV}}\right)^{\frac{3}{2}}\left(\frac{10^{12} \mathrm{GeV}}{F_{\mathcal{A}}}\right)
$$

where $\xi$ is defined as $\xi \equiv \sqrt{\left(1+|\kappa|^{2}\right) / 2}$. Using Eqs. (131) and (34), the LSP relic density is given by

$$
\Omega_{\chi} h^{2} \simeq 0.23\left(\frac{10.75}{g_{* d}}\right)^{\frac{1}{4}}\left(\frac{10^{-7} \mathrm{GeV}^{-2}}{\langle\sigma v\rangle}\right)\left(\frac{m_{\chi}}{100 \mathrm{GeV}}\right)\left(\frac{1 \mathrm{TeV}}{m_{\sigma}}\right)^{\frac{3}{2}}\left(\frac{F_{\mathcal{A}}}{10^{12} \mathrm{GeV}}\right)
$$

as long as the saxion decay provides a sufficient amount of the LSP.

The initial LSP abundance produced from the saxion decay depends on the saxion density at the decay as well as the branching ratio of the gaugino production. Since the saxion potential is generally flat, it can develop a large expectation value during inflation like a modulus field. When the Hubble parameter becomes comparable to the saxion mass, it starts to oscillate around the potential minimum with an amplitude $\sigma_{i}$. The cosmological abundance of the saxion thus depends on the initial displacement from the potential minimum, which is expected to fall in the range from $F_{\mathcal{A}}$ to $M_{P}$. The saxion dominates the the energy density of the universe if the following condition is satisfied:

$$
\frac{\sigma_{i}}{M_{P}}>\sqrt{6}\left(\frac{T_{d}}{T_{R}}\right)^{\frac{1}{2}}
$$

where $T_{R}$ is the reheating temperature, and we have assumed that the reheating is not complete when the saxion starts to oscillate. If the saxion dominates the universe, the saxion-to-entropy ratio at the decay is given by

$$
Y_{\sigma} \simeq \frac{3}{4} \frac{T_{d}}{m_{\sigma}}=8.5 \times 10^{-5} \xi\left(\frac{10.75}{g_{* d}}\right)^{\frac{1}{4}}\left(\frac{m_{\sigma}}{1 \mathrm{TeV}}\right)^{\frac{1}{2}}\left(\frac{10^{12} \mathrm{GeV}}{F_{\mathcal{A}}}\right),
$$

independent of the initial amplitude $\sigma_{i}$. The initial LSP abundance is related to $Y_{\sigma}$ as $Y_{\chi}\left(T_{d}\right)=N_{\chi} Y_{\sigma}$, where $N_{\chi}$ is the averaged number of the LSP produced by the decay of one saxion field. Since $N_{\chi}$ is close to unity from Eq. (26),$Y_{\chi}\left(T_{d}\right)$ easily exceeds the critical value $Y_{\chi}^{(c)}$, and therefore the relic LSP abundance saturates to the value given by (31).

Even when the saxion field does not dominate the universe before its decay, the LSP coming from the saxion decay tends to exceed the critical abundance $Y_{\chi}^{(c)}$. Since the reheating 
temperature is then bounded above due to the gravitino problem [27] ${ }^{\mathrm{g}}$, the saxion is likely to start to oscillate before the reheating process completes. This is the case if $T_{R}<T_{\sigma}$, where $T_{\sigma}$ is defined by

$$
T_{\sigma}=2.3 \times 10^{10} \mathrm{GeV}\left(\frac{200}{g_{* o s c}}\right)^{1 / 4}\left(\frac{m_{\sigma}}{1 \mathrm{TeV}}\right)^{1 / 2} .
$$

Here $g_{* \text { osc }}$ counts the effective number of massless degrees of freedom at $H=m_{\sigma}$. The saxion abundance is

$$
Y_{\sigma} \simeq 2.1 \times 10^{-11}\left(\frac{1 \mathrm{TeV}}{m_{\sigma}}\right)\left(\frac{T_{R}}{10^{6} \mathrm{GeV}}\right)\left(\frac{\sigma_{i}}{F_{\mathcal{A}}}\right)^{2}\left(\frac{F_{\mathcal{A}}}{10^{12} \mathrm{GeV}}\right)^{2} .
$$

Thus even if the saxion does not dominate the universe, (30) is satisfied for $\sigma_{i} \gtrsim F_{\mathcal{A}}$. So, in the following, we adopt Eq. (35) to estimate the relic LSP abundance, assuming that (30) is satisfied. Note that it is the large branching ratio into the gauginos that enables the saxion to non-thermally produce a right amount of the LSP DM, even if it does not dominate the universe.

In Fig. 1, we show the contours of the LSP abundance in $\left(m_{\sigma}, F_{\mathcal{A}}\right)$ plane. We have chosen the wino LSP with $m_{\chi}=300 \mathrm{GeV}$ and $\xi=1$ as representative values. Since the gaugino branching ratio is generically $O(1)$ as long as $m_{\sigma}>2 m_{\chi}$, the relic LSP abundance is given by Eq. (35). From the observation of SN1987A [20, 21], $F_{\mathcal{A}}$ is bounded from below, $F_{\mathcal{A}}>10^{9}$ $\mathrm{GeV}$, while an upper bound, $F_{A}<10^{12} \mathrm{GeV}$, is set by taking account of an overclosure limit of the relic axion abundance [22, 23]. We find that the observed DM abundance, $\Omega_{\chi} h^{2} \sim 0.1$, is realized for $m_{\sigma}$ around $O\left(10^{2-3}\right) \mathrm{GeV}$ and $F_{\mathcal{A}}=10^{10-12} \mathrm{GeV}$ (below the dashed line). Note that the axion overclosure bound can be relaxed by the entropy production associated with the saxion decay. In fact, in deriving the upper bound $F_{\mathcal{A}}<10^{12} \mathrm{GeV}$, it is assumed that the initial displacement of the axion from the true minimum is $O(1)$, and that there is no entropy production [28] after the axion starts to oscillate at $T=\Lambda_{Q C D} \simeq 200 \mathrm{MeV}$. If the saxion dominates the universe and decays after the QCD phase transition (of course, but before the BBN begins [29, 30] ), the upper bound can be relaxed to $\sim 10^{15} \mathrm{GeV}[24]$. Then although it depends on the initial VEVs of the axion and saxion fields, the region above the dashed line in Fig. 1] can be viable, and the right amount of the wino DM is realized even

\footnotetext{
$\mathrm{g}$ Throughout this paper we assume that there is no source for the late-time entropy production [28] other than the saxion.
} 


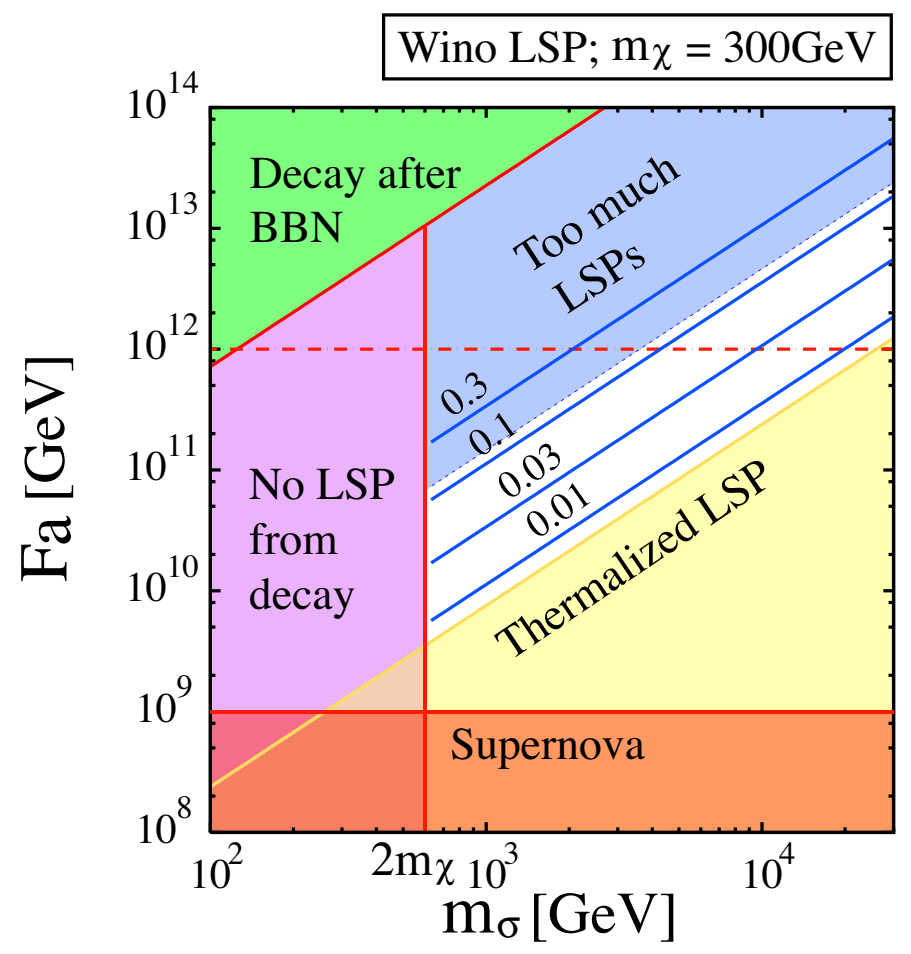

FIG. 1: Contours of the wino LSP abundance, $\Omega_{\chi} h^{2}=0.3,0.1,0.03,0.01$, for $m_{\chi}=300 \mathrm{GeV}$. The shaded regions are excluded; the LSPs are only thermally produced and therefore too small relic density for $m_{\sigma}<2 m_{\chi}$; the upper left triangle region is excluded since the saxion decays after the BBN starts, i.e., $T_{d}<5 \mathrm{MeV}$, while the lower right one corresponds to the decay temperature higher than the decoupling temperature of the wino, i.e., $T_{d}>m_{\chi} / 20 ; \Omega_{\chi} h^{2}>0.13$ is from the LSP overproduction in the light of WMAP; $F_{\mathcal{A}}<10^{9} \mathrm{GeV}$ from the SN bound. Also the region above the dashed line is disfavored by the axion overclosure limit if no entropy production occurs after the QCD phase transition.

for that region $\mathrm{h}$.

In the above numerical analyses, we have fixed the wino mass as $m_{\chi}=300 \mathrm{GeV}$. In fact, the larger LSP mass favors smaller $F_{\mathcal{A}}$ for fixed $m_{\sigma}$. This can be understood as follows; higher saxion decay temperature is needed to realize $\Omega_{\chi} h^{2} \sim 0.1$, since larger $m_{\chi}$ suppresses the annihilation cross section (see Eq. (32)). It is interesting to see that for $m_{\chi} \gtrsim 500 \mathrm{GeV}$, the

\footnotetext{
$\mathrm{h}$ The DM abundance includes the contribution from the relic axion as well as the wino/higgsino LSP. When we take into account the axion contribution, the smaller LSP abundance tends to be favored, though the result depends on both the initial displacement of the axion and the thermal history of the universe.
} 
parameter region to reproduce $\Omega_{\chi} h^{2} \sim 0.1$ (calculated by using Eq. (35)) overlaps with that where the LSP is thermalized, namely, $T_{d} \gtrsim m_{\chi} / 20$. This means that the saxion decay takes place before the decoupling of the LSP. Then we need to numerically solve the Boltzmann equation around the LSP decoupling, taking account of the LSP production from the saxion decay in order to estimate the LSP abundance correctly. Since the relic abundance depends sensitively on the evolution around the decoupling temperature [31], we do not go into details in this paper. On the other hand, the lighter LSP may be excluded by the BBN. The ${ }^{6} \mathrm{Li}$ can be overproduced due to the energetic hadrons from the DM annihilation. Thus a mass of $m_{\chi} \lesssim 100-200 \mathrm{GeV}$ is disfavored for wino/higgsino DM [32].

So far we have considered the wino LSP. When the LSP is the higgsino, $\Omega_{\chi} h^{2} \sim 0.1$ requires higher decay temperature. In fact, since the annihilation cross section is lowered by $\sim 1 / 10$, the decay temperature should be raised by about one order of magnitude. Then the constant contours of the LSP abundance in Fig. 1] shift to lower $F_{\mathcal{A}}$ by $\sim 1 / 10$ for fixed $m_{\sigma}$. Therefore, we find that the observed relic abundance of the higgsino DM can be naturally realized by the non-thermal production from the saxion decay, for $m_{\sigma}$ at the weak scale and $F_{\mathcal{A}}=10^{9-12} \mathrm{GeV}$.

In the above discussions, we have considered the hadronic axion model. Let us now comment on the DFSZ axion model. In this case the standard model particles have nonzero PQ charges, and the saxion can dominantly decay into the third generation quarks. Then the total decay rate of the saxion becomes slightly higher and $N_{\chi}$ is suppressed by that amount. It should be noted, however, that the relic DM abundance is given by Eq. (35) as long as (30) is satisfied. Therefore the contours of the LSP abundance in Fig. 1 slightly move upward for the DFSZ axion model, but our main conclusion remains virtually intact.

Lastly let us comment on the gravitino production in the saxion decay. If $\mathcal{A}$ has a mixing with the SUSY breaking field $z$, the saxion can decay into a pair of the gravitinos, in addition to the single gravitino production. In contrast to the modulus, however, the saxion decay is free from the gravitino overproduction problem. This is because the axion decay constant is much smaller than the Planck scale. Since the total decay rate is enhanced by lowering the cutoff scale, the saxion mass needs not be larger than the gravitino mass to decay before BBN, and the gravitino production may be kinematically forbidden. Even when the channel is kinematically allowed, the branching ratio of the gravitino production can be suppressed. Let us first consider the gravitino pair production rate. Since the coupling of 
$\mathcal{A}$ with the two gravitinos is proportional to $F_{\mathcal{A}}[10]$, the branching ratio of the gravitino production is suppressed by $\left(F_{\mathcal{A}} / M_{P}\right)^{4}$, namely $\kappa_{3 / 2}=O\left(F_{\mathcal{A}} / M_{P}\right)^{4}$. Since $F_{\mathcal{A}}$ is bounded above, $F_{\mathcal{A}} \lesssim 10^{15} \mathrm{GeV}$, leading to $\kappa_{3 / 2} \sim 10^{-12}$ which is small enough to evade the bounds from the BBN and LSP overclosure limits [6]. However, the single-gravitino production rate actually dominates over the pair production rate, if the saxion mass is larger than $m_{3 / 2}$ due to the non-SUSY mass term. The branching ratio of the single-gravitino production is given by $O\left(\left(m_{\phi} / m_{3 / 2}\right)^{2}\left(F_{\mathcal{A}} / M_{P}\right)^{2}\right)$, and the cosmological bounds are not so severe unless $m_{\phi} \gg m_{3 / 2}$. Note that, since the saxion does not necessarily dominate the universe to produce a right amount of the wino/higgsino DM, the gravitino abundance from the saxion decay may be suppressed. Therefore the gravitino production can be safely neglected in the saxion decay.

\section{CONCLUSIONS AND DISCUSSION}

In this paper we have pointed out that an intermediate scale physics is necessary for a successful non-thermal production of the wino/higgsino DM, to avoid the moduli-induced gravitino/LSP problem recently pointed out in Refs. 6]. This is because gravitinos and/or LSPs would be overproduced otherwise. The conventional scenario using the modulus decay to produce the wino/higgsino DM non-thermally is disfavored from this point of view. Instead we have proposed an alternative candidate: the bosonic SUSY partner of the axion, i.e., the saxion. It is particularly interesting that the right amount of the DM can be realized for the saxion mass around the weak scale when we consider the axion decay constant within the cosmologically allowed range, $F_{\mathcal{A}}=10^{9-12} \mathrm{GeV}$. Although our analyses focused on the case of the saxion, we would like to stress that they are rather generic and can be applied to any scalar fields that decay via the non-renormalizable operators of $D=5$ with an intermediate cut-off scale.

It is also worth noting that the saxion decay is applicable for a source of the harmless entropy production. As was stressed in this paper, the late-time decay of the scalar field generally suffers from the gravitino/LSP overproduction. In contrast, the scalar field at an intermediate scale can reheat the universe avoiding overproduction of the gravitino/superparticles. For instance, a scalar field at $M \ll M_{P}$ may decay before BBN begins, while satisfying $m_{\phi}<2 m_{3 / 2}, 2 m_{\mathrm{LSP}}$ to kinematically block the gravitino/LSP production 
channels.

\section{acknowledgement}

We are grateful to J.P. Conlon and M. Senami for comments. The authors would like to thank the Japan Society for Promotion of Science for financial support.

[1] L. Randall and R. Sundrum, Nucl. Phys. B557, 79 (1999);

G.F. Giudice, M.A. Luty, H. Murayama and R. Rattazzi, JHEP 9812, 027 (1998);

J.A. Bagger, T. Moroi and E. Poppitz, JHEP 0004, 009 (2000).

[2] M. Endo, M. Yamaguchi and K. Yoshioka, Phys. Rev. D 72, 015004 (2005);

K. Choi, K. S. Jeong and K. i. Okumura, JHEP 0509, 039 (2005);

A. Falkowski, O. Lebedev and Y. Mambrini, JHEP 0511, 034 (2005).

[3] Recently by R. Kitano and Y. Nomura, arXiv hep-ph/0602096;

E. A. Baltz, M. Battaglia, M. E. Peskin and T. Wizansky, arXiv hep-ph/0602187

H. Baer, A. Mustafayev, E. K. Park, S. Profumo and X. Tata, arXiv hep-ph/0603197;

G. B. Gelmini and P. Gondolo, arXiv hep-ph/0602230

G. Gelmini, P. Gondolo, A. Soldatenko and C. E. Yaguna, arXiv hep-ph/0605016.

See also references therein.

[4] D. N. Spergel et al., arXiv:astro-ph/0603449.

[5] T. Moroi and L. Randall, Nucl. Phys. B 570, 455 (2000).

[6] M. Endo, K. Hamaguchi and F. Takahashi, Phys. Rev. Lett. 96, 211301 (2006);

S. Nakamura and M. Yamaguchi, arXiv hep-ph/0602081.

[7] M. Kawasaki, F. Takahashi and T. T. Yanagida, arXiv hep-ph/0603265 arXiv:hep-ph/0605297.

[8] T. Asaka, S. Nakamura and M. Yamaguchi, arXiv hep-ph/0604132.

[9] M. Dine, R. Kitano, A. Morisse and Y. Shirman, arXiv hep-ph/0604140.

[10] M. Endo, K. Hamaguchi and F. Takahashi, arXiv hep-ph/0605091.

[11] S. Khalil, C. Munoz and E. Torrente-Lujan, New J. Phys. 4, 27 (2002).

[12] M. Fujii and K. Hamaguchi, Phys. Lett. B 525, 143 (2002); Phys. Rev. D 66, 083501 (2002). 
[13] N.V. Krasnikov, Phys. Lett. B193 37 (1987); T.R. Taylor, Phys. Lett. B252 59 (1990); J.A. Casas, Z. Lalak, C. Munoz and G.G. Ross, Nucl. Phys. B347 243 (1990); B. de Carlos, J.A. Casas and C. Munoz, Nucl. Phys. B399 623 (1993).

[14] K. Kohri, M. Yamaguchi and J. Yokoyama, Phys. Rev. D 70, 043522 (2004); Phys. Rev. D 72, 083510 (2005).

[15] W. Buchmuller, K. Hamaguchi, M. Ratz and T. Yanagida, Phys. Lett. B 588, 90 (2004).

[16] R. D. Peccei and H. R. Quinn, Phys. Rev. Lett. 38, 1440 (1977).

[17] F. Wilczek, Phys. Rev. Lett. 40, 279 (1978).

[18] J. E. Kim, Phys. Rev. Lett. 43, 103 (1979);

M. A. Shifman, A. I. Vainshtein and V. I. Zakharov, Nucl. Phys. B 166, 493 (1980).

[19] M. Dine, W. Fischler and M. Srednicki, Phys. Lett. B 104, 199 (1981);

A. R. Zhitnitsky, Sov. J. Nucl. Phys. 31, 260 (1980) [Yad. Fiz. 31, 497 (1980)].

[20] G. G. Raffelt, Stars as laboratories for fundamental physics: The astrophysics of neutrinos, axions, and other weakly interacting particles. The University of Chicago Press, Chicago \& London, 1996.

[21] E. W. Kolb and M. S. Turner, The Early Universe. Addison-Wesley, Redwood City, USA, 1990.

[22] J. Preskill, M. B. Wise and F. Wilczek, Phys. Lett. B 120, 127 (1983);

L. F. Abbott and P. Sikivie, Phys. Lett. B 120, 133 (1983);

M. Dine and W. Fischler, Phys. Lett. B 120, 137 (1983).

[23] M. S. Turner, Phys. Rev. D 33, 889 (1986).

[24] M. Kawasaki, T. Moroi and T. Yanagida, Phys. Lett. B 383, 313 (1996).

[25] M. Hashimoto, K. I. Izawa, M. Yamaguchi and T. Yanagida, Phys. Lett. B 437, 44 (1998).

[26] K. A. Olive and M. Srednicki, Phys. Lett. B 230, 78 (1989).

[27] M. Kawasaki, K. Kohri and T. Moroi, Phys. Lett. B 625, 7 (2005); Phys. Rev. D 71, 083502 (2005).

[28] D. H. Lyth and E. D. Stewart, Phys. Rev. D 53, 1784 (1996);

M. Kawasaki and F. Takahashi, Phys. Lett. B 618, 1 (2005).

[29] M. Kawasaki, K. Kohri and N. Sugiyama, Phys. Rev. Lett. 82, 4168 (1999);

Phys. Rev. D 62, 023506 (2000);

K. Ichikawa, M. Kawasaki and F. Takahashi, Phys. Rev. D 72, 043522 (2005). 
[30] S. Hannestad, Phys. Rev. D 70, 043506 (2004).

[31] M. Drees, H. Iminniyaz and M. Kakizaki, arXiv hep-ph/0603165.

[32] K. Jedamzik, Phys. Rev. D 70, 083510 (2004). 